\title{
INFLUENCE OF NON-LINEAR PARAMETRIC EXCITATION ON RESONANT CHARACTERISTICS OF OSCILLATING SYSTEMS
}

\author{
NGUYEN VAN KHANG - TRAN DINH SON \\ Hanoi University of Technology
}

\section{Introduction}

One of the most important tasks of oscillation investigation in engineering is to determine resonant regimes. Several problems related to parametric excitation have been quite thoroughly examined in $[1,2]$. The objective of this paper is to examine the influence of non-linear parametric excitation to resonant characteristics of one degree of freedom system in which non-linear function contains derivatives of second order $[3,4]$.

2. Averaging method for a system with second order derivative in the right side

Consider an oscillating system described by the differential equation

$$
\ddot{x}+n^{2} x+f(\tau)=\varepsilon F(\tau, x, \dot{x}, \ddot{x})
$$

where $f(\tau), F(\tau, x, \dot{x}, \ddot{x})$ are periodic functions of $\tau$ with the period $2 \pi, n$ is an integer and $\varepsilon$ is a small parameter. Using the variable transformation:

$$
\begin{aligned}
& x=r(\tau) \cos n \tau+s(\tau) \sin n \tau+x^{*}(\tau)^{*} \\
& \dot{x}=-r(\tau) n \sin n \tau+s(\tau) n \cos n \tau+\dot{x}^{*}(\tau)
\end{aligned}
$$

where $r$ and $s$ are new variables,

$$
\begin{gathered}
f(\tau)=\frac{a_{0}}{2}+\sum_{i=1}^{\infty}\left(a_{j} \cos j \tau+b_{j} \sin j \tau\right), \\
x^{*}(\tau)=-\frac{a_{0}}{2 n^{2}}-\sum_{\substack{j=1 \\
j \neq n}}^{\infty} \frac{a_{j} \cos j \tau+b_{j} \sin j \tau}{n^{2}-j^{2}} .
\end{gathered}
$$


Equations for new variables will be

$$
\begin{aligned}
\frac{d r}{d \tau}= & -\frac{\varepsilon}{n} F\left(\tau, x^{*}+r \cos n \tau+s \sin n \tau, \dot{x}^{*}-r n \sin n \tau+s n \cos n \tau,\right. \\
& \left.\ddot{x}^{*}-r n^{2} \cos n \tau-s n^{2} \sin n \tau-\dot{r} n \sin n \tau+\dot{s} n \cos n \tau\right) \sin n \tau, \\
\frac{d s}{d \tau}= & \frac{\varepsilon}{n} F\left(\tau, x^{*}+r \cos n \tau+s \sin n \tau, \dot{x}^{*}-r n \sin n \tau+s n \cos n \tau,\right. \\
& \left.\ddot{x}^{*}-r n^{2} \cos n \tau-s n^{2} \sin n \tau-\dot{r} n \sin n \tau+\dot{s} n \cos n \tau\right) \cos n \tau .
\end{aligned}
$$

The averaging method can be applied to the equations $(2.4)$ to find out its approximate solutions [4]. First we write the equations (2.4) in the form:

$$
\begin{aligned}
\frac{d r}{d t}= & -\frac{\varepsilon}{n} F\left(\tau, x^{*}+r \cos n \tau+s \sin n \tau, \dot{x}^{*}-r n \sin n \tau+s n \cos n \tau,\right. \\
& \left.\ddot{x}^{*}-r n^{2} \cos n \tau-s n^{2} \sin n \tau\right) \sin n \tau, \\
\frac{d s}{d t}= & \frac{\varepsilon}{n} F\left(\tau, x^{*}+r \cos n \tau+s \sin n \tau, \dot{x}^{*}-r n \sin n \tau+s n \cos n \tau,\right. \\
& \left.\ddot{x}^{*}-r n^{2} \cos n \tau-s n^{2} \sin n \tau\right) \cos n \tau .
\end{aligned}
$$

Averaging the right hand sides of these equations we obtain

$$
\begin{gathered}
\frac{d r}{d \tau}=-\frac{\varepsilon}{n}\langle F \sin n \tau\rangle=\varepsilon \phi_{1}(r, s), \\
\frac{d s}{d \tau}=\frac{\varepsilon}{n}\langle F \cos n \tau\rangle=\varepsilon \phi_{2}(r, s) \\
\langle f\rangle=\frac{1}{2 \pi} \int_{0}^{2 \pi} f d \tau
\end{gathered}
$$

In general, it is impossible to integrate the equation (2.6). However, we can find its important particular solution $r_{0}=$ const, $s_{0}=$ const, which satisfies equations

$$
\begin{aligned}
& \phi_{1}\left(r_{0}, s_{0}\right)=0 \\
& \phi_{2}\left(r_{0}, s_{0}\right)=0 .
\end{aligned}
$$

The equation system (2.8) is called amplitude equations since $A=\sqrt{r^{2}+s^{2}}$ is the amplitude of oscillation. The solution of equation (2.1) that corresponds to $\left(r_{0}, s_{0}\right)$ is called the stationary one. 
In order to study the stability of the solution $\left(r_{0}, s_{0}\right)$ of the equation (2.6) we consider an arbitrary solution $(r, s)$, whose initial values are close to $\left(r_{0}, s_{0}\right)$ :

$$
\begin{aligned}
& r=r_{0}+\delta r \\
& s=s_{0}+\delta s .
\end{aligned}
$$

Substitution of (2.9) into (2.6) and development of their right members into power series of $\delta r, \delta s$ yield

$$
\begin{aligned}
& \frac{d(\delta r)}{d \tau}=\varepsilon\left(\frac{\partial \phi_{1}}{\partial r}\right)_{0} \delta r+\varepsilon\left(\frac{\partial \phi_{1}}{\partial s}\right)_{0} \delta s+\ldots \\
& \frac{d(\delta s)}{d \tau}=\varepsilon\left(\frac{\partial \phi_{2}}{\partial r}\right)_{0} \delta r+\varepsilon\left(\frac{\partial \phi_{2}}{\partial s}\right)_{0} \delta s+\ldots
\end{aligned}
$$

where non-written terms are of higher order than one relatively to $\delta r$ and $\delta s$ and the symbols $(. .)_{0}$ denotes that after differentiating we put $r=r_{0}, s=s_{0}$.

Necessary and sufficient conditions for asymptotic stability of stationary solution are

$$
\begin{array}{r}
\left(\frac{\partial \phi_{1}}{\partial r}\right)_{0}+\left(\frac{\partial \phi_{2}}{\partial s}\right)_{0}<0 \\
\left(\frac{\partial \phi_{1}}{\partial r} \cdot \frac{\partial \phi_{2}}{\partial s}-\frac{\partial \phi_{1}}{\partial s} \cdot \frac{\partial \phi_{2}}{\partial r}\right)_{0}>0
\end{array}
$$

\section{Amplitude-Frequency Equation of system with non-lin- ear parametric excitation term}

Examine resonant oscillations of an one degree of freedom system given by the equation:

$$
\ddot{x}+\Lambda x=\varepsilon\left(\Lambda \alpha x+\sum_{j=1}^{5} g_{j} x^{j} \cos m \tau-\beta \dot{x}+e x^{3}+e_{1} x \dot{x}^{2}+e_{2} x^{2} \ddot{x}\right)
$$

where the overdots denote derivation with respect to variable $\tau$ and $m$ is an integer. In this case the expression $F(\tau, x, \dot{x}, \ddot{x})$ is of the form

$$
F=\Lambda \alpha x+\sum_{j=1}^{5} g_{j} x^{j} \cos m \tau-\beta \dot{x}+e x^{3}+e_{1} x \dot{x}^{2}+e_{2} x^{2} \ddot{x}
$$

Equation (3.1) can be now rewritten under the form:

$$
\ddot{x}+\Lambda x=\varepsilon F(\tau, x, \dot{x}, \ddot{x}) .
$$


Consider the resonant case $\Lambda=n^{2}$. In the first approximation the solution of he equation (3.1) is assumed of the form

$$
\begin{aligned}
& x=r \cos n \tau+s \sin n \tau \\
& \dot{x}=-r n \sin n \tau+s n \cos n \tau \\
& \ddot{x}=-r n^{2} \cos n \tau-s n^{2} \sin n \tau .
\end{aligned}
$$

Jubstituting (3.3) into (2.6) and making some transformations, the following amplitude - frequency equation of stationary oscillation can be established

$$
\begin{aligned}
& \left(n^{2} \alpha+\frac{3 e+n^{2} e_{1}-3 n^{2} e_{2}}{4} A^{2}\right)\left(\begin{array}{c}
s \\
r
\end{array}\right)-n \beta\left(\begin{array}{c}
-r \\
s
\end{array}\right)+\frac{1}{2} g_{1} \delta_{m}^{2 n}\left(\begin{array}{c}
-s \\
r
\end{array}\right) \\
& +\frac{g_{2}}{4} \delta_{m}^{n}\left(\begin{array}{c}
2 r s \\
3 r^{2}+s^{2}
\end{array}\right)+\frac{g_{2}}{4} \delta_{m}^{3 n}\left(\begin{array}{c}
-2 r s \\
r^{2}-s^{2}
\end{array}\right)+\frac{g_{3}}{2} \delta_{m}^{2 n}\left(\begin{array}{c}
-s^{3} \\
r^{3}
\end{array}\right) \\
& +\frac{g_{3}}{8} \delta_{m}^{4 n}\left(\begin{array}{c}
s^{3}-3 r^{2} s \\
r^{3}-3 r s^{2}
\end{array}\right)+\frac{g_{4} A^{2}}{8} \delta_{m}^{n}\left(\begin{array}{c}
4 r s \\
5 r^{2}+s^{2}
\end{array}\right)+\frac{g_{4}}{16} \delta_{m}^{3 n}\left(\begin{array}{c}
-4 s r^{3}-12 r s^{3} \\
5 r^{4}-3 s^{4}-6 r^{2} s^{2}
\end{array}\right) \\
& +\frac{g_{4}}{16} \delta_{m}^{5 n}\left(\begin{array}{c}
-4 s r^{3}+4 r s^{3} \\
r^{4}+s^{4}-6 r^{2} s^{2}
\end{array}\right)+\frac{5 g_{5} A^{2}}{32} \delta_{m}^{2 n}\left(\begin{array}{c}
s r^{2}-3 s^{3} \\
3 r^{3}-r s^{2}
\end{array}\right) \\
& +\frac{g_{5}}{32} \delta_{m}^{4 n}\left(\begin{array}{c}
6 s^{5}-10 s r^{4}-20 r^{2} s^{3} \\
6 r^{5}-10 r s^{4}-20 r^{3} s^{2}
\end{array}\right)+\frac{g_{5}}{32} \delta_{m}^{6 n}\left(\begin{array}{c}
-s^{5}-5 s r^{4}+10 r^{2} s^{3} \\
r^{5}+5 r s^{4}-10 r^{3} s^{2}
\end{array}\right)=0,
\end{aligned}
$$

where

$$
\delta_{i}^{j}= \begin{cases}0 & \text { for } j \neq i \\ 1 & \text { for } j=i\end{cases}
$$

Equation (3.4) always has a trivial solution $A=0$. For $A \neq 0$ it follows from equation (3.4) a formula relating amplitude to frequency. The results obtained are recapitulated in the Table 1.

\section{Stability Analysis of Resonant Oscillations}

With the help of stability conditions (2.11), (2.12) we can establish stability conditions for stationary solutions of the equation (3.1) of the parameter oscillation. Final results obtained after rather complicated transformations are as follows: 


\begin{tabular}{|c|c|}
\hline $\begin{array}{l}\text { Resonance } \\
\text { possibilities }\end{array}$ & Amplitude - frequency formulas \\
\hline$m=n$ & $\begin{aligned} n^{2} \alpha=- & \frac{3 e+n^{2} e_{1}-3 n^{2} e_{2}}{4} A^{2} \pm \\
& \frac{6 g_{2}+5 g_{4} A^{2}}{2 g_{2}+g_{4} A^{2}} \sqrt{\frac{\left(2 g_{2}+g_{4} A^{2}\right)^{2} A^{2}}{64}-n^{2} \beta^{2}}\end{aligned}$ \\
\hline$m=2 n$ & $\begin{array}{c}n^{2} \alpha=-\frac{3 e+n^{2} e_{1}-3 n^{2} e_{2}}{4} A^{2} \pm \frac{16 g_{1}+16 g_{3} A^{2}+15 g_{5} A^{4}}{16 g_{1}+8 g_{3} A^{2}+5 g_{5} A^{4}} \\
\times \sqrt{\left(\frac{16 g_{1}+8 g_{3} A^{2}+5 g_{5} A^{4}}{32}\right)^{2}-n^{2} \beta^{2}}\end{array}$ \\
\hline$m=3 n$ & $\begin{aligned} n^{2} \alpha=- & \frac{3 e+n^{2} e_{1}-3 n^{2} e_{2}}{4} A^{2} \pm \\
& \frac{4 g_{2}+5 g_{4} A^{2}}{4 g_{2}+3 g_{4} A^{2}} \sqrt{\frac{\left(4 g_{2}+3 g_{4} A^{2}\right)^{2} A^{2}}{16^{2}} \cdot-n^{2} \beta^{2}}\end{aligned}$ \\
\hline$m=4 n$ & $\begin{aligned} n^{2} \alpha=- & \frac{3 e+n^{2} e_{1}-3 n^{2} e_{2}}{4} A^{2} \pm \\
& \frac{2 g_{3}+3 g_{5} A^{2}}{2 g_{3}+2 g_{5} A^{2}} \sqrt{\frac{\left(g_{3}+g_{5} A^{2}\right)^{2} A^{4}}{64}-n^{2} \beta^{2}}\end{aligned}$ \\
\hline$m=5 n$ & $n^{2} \alpha=-\frac{3 e+n^{2} e_{1}-3 n^{2} e_{2}}{4} A^{2} \pm \sqrt{\frac{g_{4}^{2} A^{6}}{16^{2}}-n^{2} \beta^{2}}$ \\
\hline$m=6 n$ & $n^{2} \alpha=-\frac{3 e+n^{2} e_{1}-3 n^{2} e_{2}}{4} A^{2} \pm \sqrt{\frac{g_{5}^{2} A^{8}}{32^{2}}-n^{2} \beta^{2}}$ \\
\hline
\end{tabular}

a. Case $m=n$

For stationary solution $A \neq 0$ stability conditions are

$$
\frac{d \alpha}{d A}<0 \text { or } \frac{d \alpha}{d A}>0
$$

for the sign before the symbol of square root in amplitude-frequency formula is positive or negative, respectively.

For the trivial solution $A=0$, stability conditions are

$$
\begin{gathered}
-\beta<0, \\
n^{2} \beta^{2}>-n^{4} \alpha^{2} .
\end{gathered}
$$


Since these conditions are always satisfied, the solution $A=0$ is stable.

b. Case $m=2 n$

For stationary solution $A \neq 0$ stability conditions are

$$
\frac{d \alpha}{d A}<0 \text { for } \frac{d \alpha}{d A}>0
$$

for the sign before the symbol of square root in amplitude-frequency formula is positive or negative, respectively.

For the trivial solution $A=0$, stability conditions are

$$
\begin{aligned}
-\beta & <0 \\
n^{2} \beta^{2} & >\frac{1}{4} g_{1}^{2}-\left(n^{2} \alpha\right)^{2},
\end{aligned}
$$

it follows that the solution $A=0$ is only stable outside resonant region.

c. Case $m=3 n$

For stationary solution $A \neq 0$ stability conditions are

$$
\frac{d \alpha}{d A}<0 \text { or } \frac{d \alpha}{d A}>0
$$

for the sign before the symbol of square root in amplitude - frequency formula is positive or negative, respectively.

For the trivial solution $A=0$, stability conditions are always satisfied, the solution $A=0$ is stable.

d. Case $m=4 n$

For stationary solution $A \neq 0$, stability conditions are

$$
\frac{d \alpha}{d A}<0 \text { or } \frac{d \alpha}{d A}>0
$$

for the sign before the symbol of square root in amplitude - frequency formula is positive or negative, respectively.

For the trivial solution $A=0$, stability conditions are always satisfied, the solution $A=0$ is stable.

e. Case $m=5 n$

For stationary solution $A \neq 0$, stability conditions are

$$
\frac{d \alpha}{d a}<0 \text { or } \frac{d \alpha}{d a}>0
$$


for the sign before the symbols of square root in amplitude - frequency formula is positive or negative, respectively.

For the trivial solution $A=0$, stability conditions are always satisfied, the solution $A=0$ is stable

f. Case $m=6 n$

For stationary solution $A \neq 0$, stability conditions are

$$
\frac{d \alpha}{d A}<0 \text { or } \frac{d \alpha}{d A}>0
$$

for the sign before the symbol of square root in amplitude - frequency formula is positive or negative, respectively.

For the trivial solution $A=0$, stability conditions are always satisfied, the solution $A=0$ is stable.

\section{Numerical Simulation}

The values of the following parameters are given for numerical simulation:

$$
\begin{array}{ll}
g_{1}=0.3 ; & g_{2}=0.1 ; g_{3}=0.03 ; g_{4}=0.02 ; g_{5}=0.008 \\
e=0.01 ; \quad e_{1}=0.02 ; \quad e_{2}=0.02 .
\end{array}
$$

The remaining data are given according to each resonant case, such as:

+ For case $m=n$, given $m=n=4 ; \beta=0.003 ; \alpha=0.05$.

+ For case $m=2 n$, given $m=6 ; \beta=0.03 ; \alpha=0$.

+ For case $m=3 n$, given $m=9 ; \beta=0.03 ; \alpha=0.05$.

+ For case $m=4 n$, given $m=16 ; \beta=0.03 ; \alpha=0.1$.

+ For case $m=5 n$, give $m=15 ; \beta=0.003 ; \alpha=0.08$.

+ For case $m=6 n$, give $m=18 ; \beta=0.003 ; \alpha=0.08$.

The MATLAB program [5] has been used for determination of amplitude frequency curve according to formulas in the Table 1 and for integration of differential equation of oscillation with initial conditions determined from the amplitude - frequency curves.

Fig. 1 represents the amplitude - frequency curves of resonant oscillations.

Fig. 2 shown graphically oscillation in time domain. It is noticed that there is a correspondence between the results obtained by analytic method and by numerical simulation and that harmonic resonant oscillations depend strongly on the damping parameter $\beta$. 
Resonant curve in the case $m=n=4$

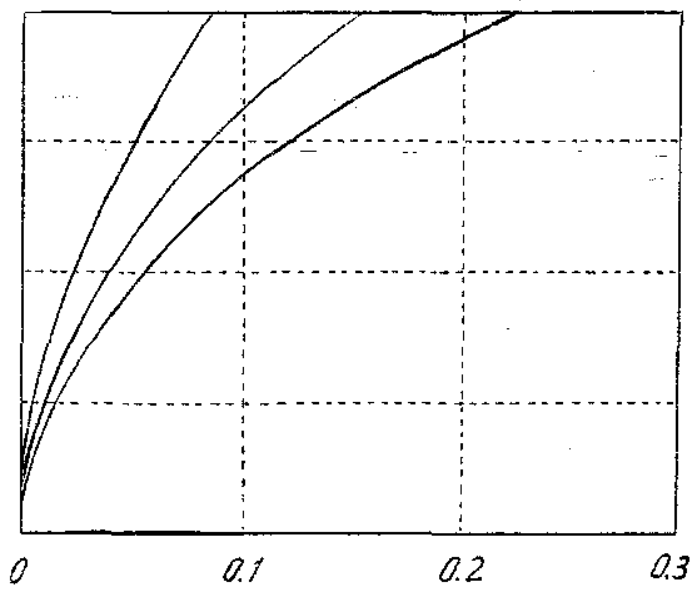

Resonant curve in the case $m=3 n=9$

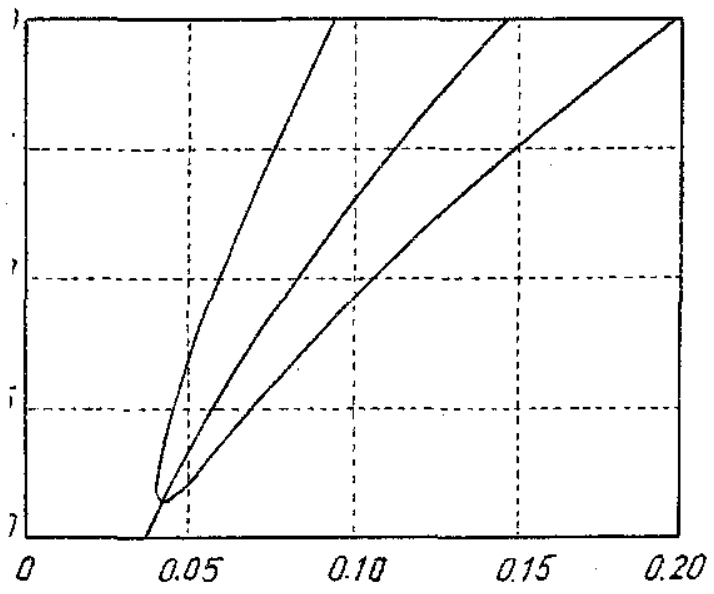

Resonant curve in the case $m=5 n=15$

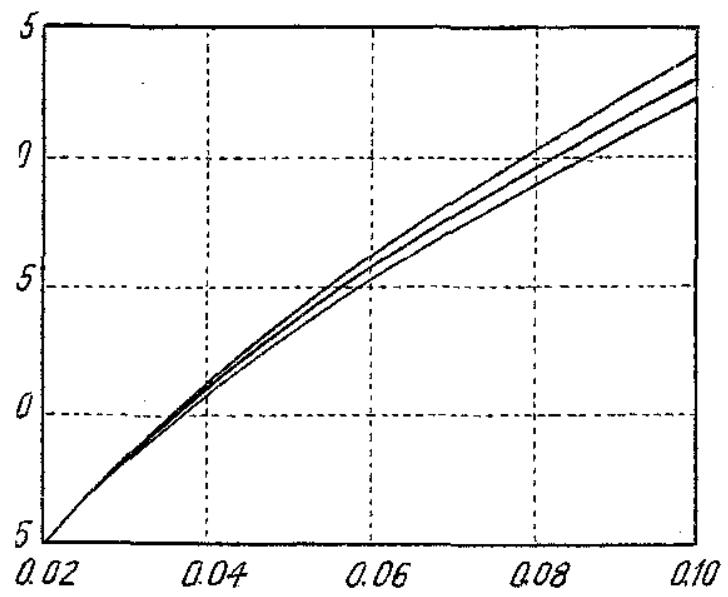

Resonant curve in the case $m=2 n=6$

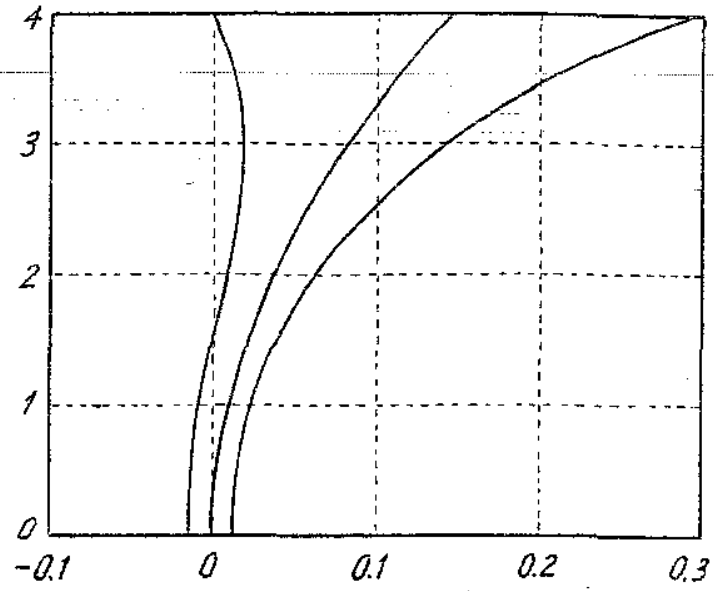

Resonant curve in the case $m=4 n=16$

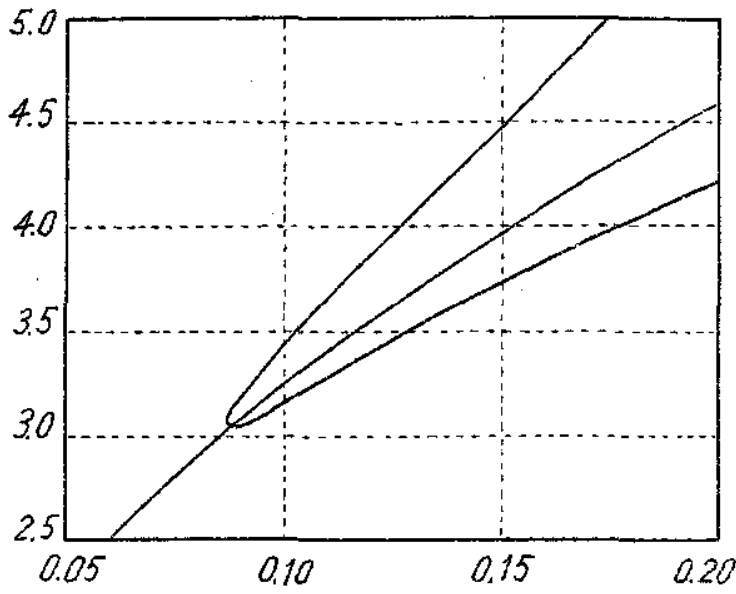

Resonant curve in the case $m=6 n=18$

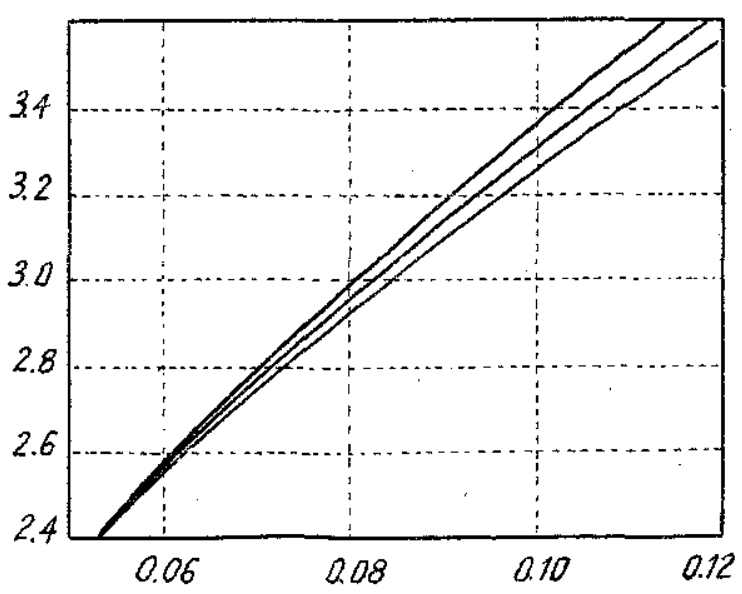

Fig. 1 

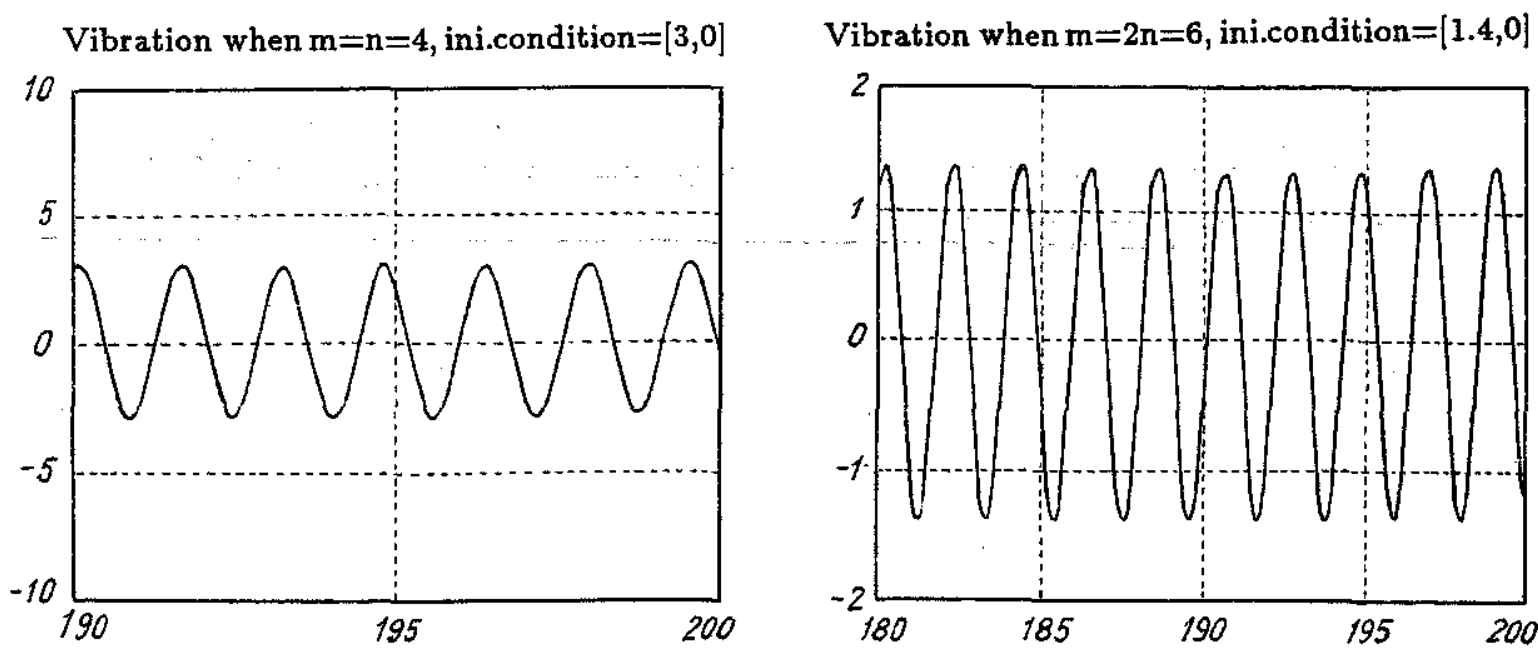

Vibration when $m=n=9$, ini. condition $=[2.6,0]$

Vibration when $m=4 n=16$, ini.condition $=[3.4,0]$
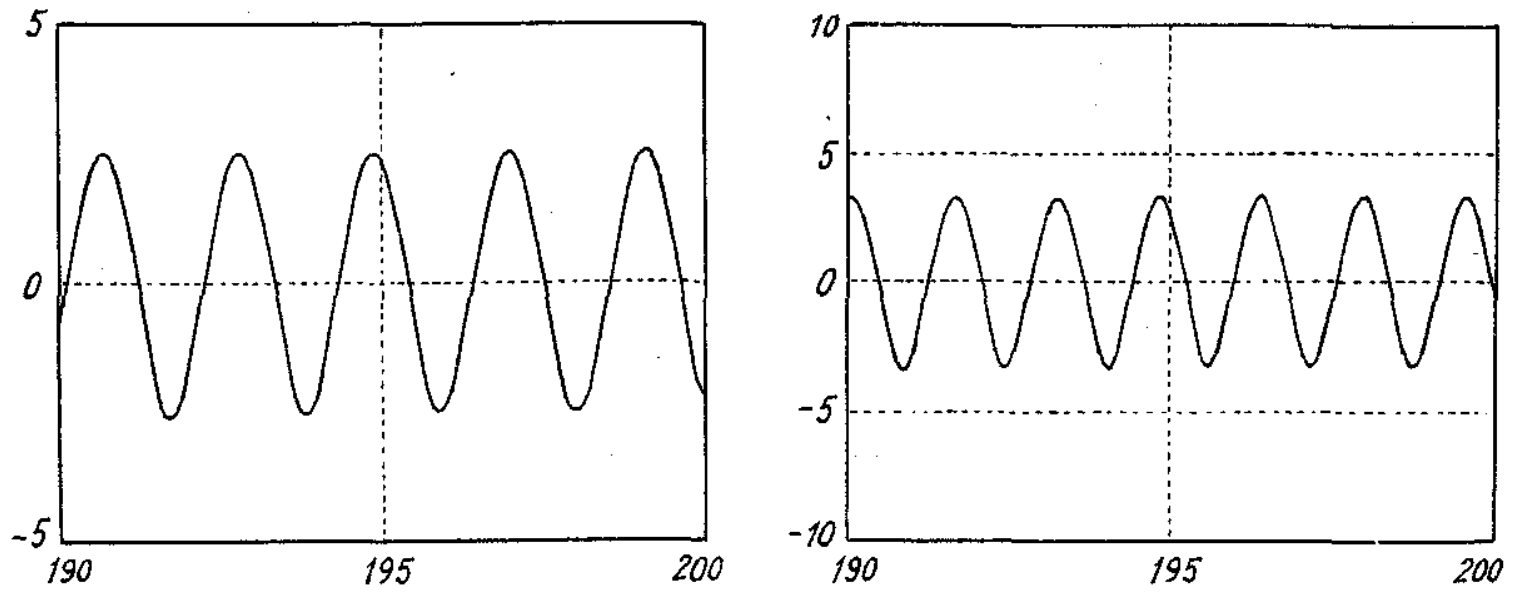

Vibration when $m=n=15$, ini.condition $=[3.0,3.0]$

Vibration when $m=n=18$, ini.condition $=[3,0]$
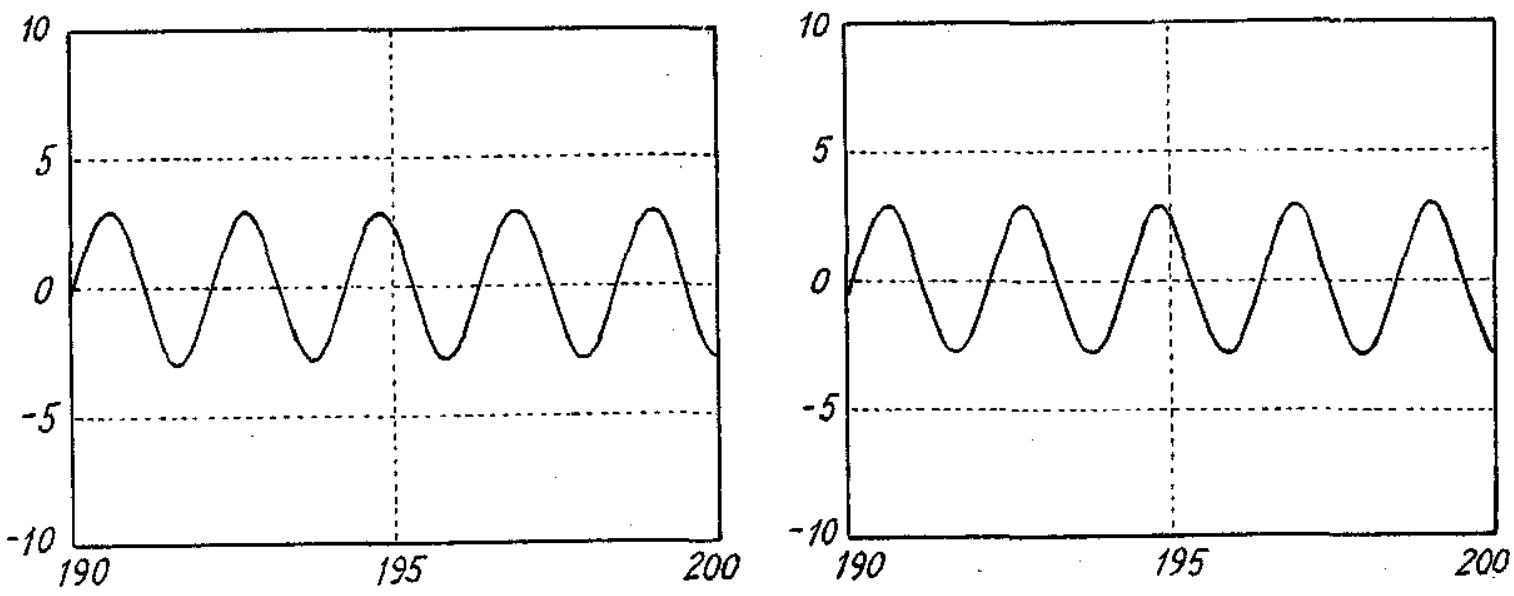

Fig. 2 


\section{Conclusion}

This article presented an application of averaging method to the examination of resonant oscillations in a system with nonlinear parametric excitation. An analytic approach has provided amplitude - frequency equations and enabled to examine stability regimes of possible resonant oscillations.

Analytic results correspond satisfactorily to the ones obtained by numerical simulation. It is shown a strong dependence of periodic oscillations on damping parameter $\beta$. Further investigation of influence of $\beta$ on oscillation regimes of the system will be published later.

This work is completed with financial support from the Council for Natural Sciences of Vietnam.

\section{References}

1. Schmidt G. Parameterregte Schwingungen. Deutscher Verlag der Wissenschaften, Berlin 1975.

2. Mitropolskii Yu. A., Nguyen Van Dao. Applied Asymptotic Methods in Nonlinear Oscillations. Kluwer Academic Publishers 1997.

3. Nguyen Van Khang. Determination of the oscillations in the systems with nonlinear inertia term by method of small parameter (in Vietnamese). Vietnam Journal of Mechanics, No.4, p. 12-18, Hanoi 1980.

4. Филатов А. Н. Методы усреднения в дифференциальных и интегро дифференциальных уравнениях. Изд. “Фан” УССР Ташкент 1971.

5. Biran A., Breiner M. MALAB fuer Ingenieure. Addison-Wesley Publishing Company, Bonn 1995.

VỀ ÂNH HƯ̛̛NG CƯA KÍCH ĐộNG THAM SỐ PHI TUYẼN

ĐẾN TÍNH CHẤT CỘNG HỪ̛̛NG CƯA HỆ DAO ĐỘNG

Trong bài báo đã áp dụng phương pháp trung bình để khảo sát dao động cộng hường của một hệ dao động có kích động tham số phi tuyến. Bằng công cụ giải tích đã thu được phương trình biên độ - tần số và khảo sát các chế độ ồn định của các dao động cộng hưởng xuất hiện. Các kết quả tính toán giải tích khá phù hợp với các kết quả tính toán bằng số. Các kết quá thu được cho thấy sự phụ thuộc rất mạnh của các nghiệm tuần hoàn vào tham số cản $\beta$. 